PROCEEDINGS OF THE

AMERICAN MATHEMATICAL SOCIETY

Volume 132, Number 9, Pages 2661-2669

S 0002-9939(04)07419-2

Article electronically published on April 8, 2004

\title{
INFINITE LIFETIME FOR THE STARLIKE DYNAMICS IN HELE-SHAW CELLS
}

\author{
BJÖRN GUSTAFSSON, DMITRI PROKHOROV, AND ALEXANDER VASIL'EV
}

(Communicated by Juha M. Heinonen)

\begin{abstract}
One of the "folklore" questions in the theory of free boundary problems is the lifetime of the starlike dynamics in a Hele-Shaw cell. We prove precisely that, starting with a starlike analytic phase domain $\Omega_{0}$, the HeleShaw chain of subordinating domains $\Omega(t), \Omega_{0}=\Omega(0)$, exists for an infinite time under injection at the point of starlikeness.
\end{abstract}

\section{INTRODUCTION}

We consider the flow of a viscous fluid in a plane Hele-Shaw cell under injection through a unique well which can be placed at the origin. Suppose that at the initial time the phase domain $\Omega_{0}$ occupied by the fluid is simply connected and bounded by a smooth analytic curve $\Gamma_{0}$. The evolution of the phase domains $\Omega(t)$, $\Omega(0)=\Omega_{0}$, is described by an auxiliary conformal mapping $f(\zeta, t), f(\zeta, 0)=f_{0}(\zeta)$, of the unit disk $U=\{\zeta,|\zeta|<1\}$ onto $\Omega(t), \Gamma(t)=\partial \Omega(t)$, normalized by $f(0, t)=0$, $f^{\prime}(0, t)>0$. Here we denote the derivatives by $f^{\prime}=\partial f / \partial \zeta, \dot{f}=\partial f / \partial t$, and $t$ is a time parameter. This mapping satisfies the equation

$$
\operatorname{Re}\left[\dot{f}(\zeta, t) \overline{\zeta f^{\prime}(\zeta, t)}\right]=1, \quad \zeta=e^{i \theta}
$$

under a suitable rescaling. L. A. Galin 4 and P. Ya. Polubarinova-Kochina [11, 12 first derived the equation (1.1) and stimulated deep investigations in the complex variable approach to free boundary problems (see, e.g., 9, 20] and the references therein).

By a classical solution in the interval $t \in[0, T)$ to the equation (1.1) we mean a map $f(\zeta, t)$ that is conformal and univalent as a function of $\zeta$ in a neighbourhood of the closure $\bar{U}$ of the unit disk $U$ and $C^{1}$ with respect to $t$ in $[0, T$ ) (one-sided at 0 ). These assumptions about the classical solution can be found, e.g., in [14.

One of the main features of the solution to the equation (1.1) is that, starting with an analytic boundary $\Gamma_{0}$, we obtain a one-parameter $(t)$ chain of classical

Received by the editors January 3, 2003 and, in revised form, June 10, 2003.

2000 Mathematics Subject Classification. Primary 30C45, 76D27, 76S05; Secondary 35Q35, $30 \mathrm{C} 35$.

Key words and phrases. Free boundary problem, Hele-Shaw flow, univalent function, starlike function, Löwner-Kufarev equation.

The first author was partially supported by the Swedish Research Council, the Göran Gustafsson Foundation, and Fondecyt (Chile) \# 7030011. The second author was supported by Fondecyt (Chile) \# 7010093, and the third author was partially supported by Projects Fondecyt (Chile) \# 1030373, 1020067, and UTFSM 12.03.23. 
solutions $f(\zeta, t)$ that exists during a period $t \in[0, T)$, developing possible cusps or double points on the boundary $\Gamma(t), \Gamma(0)=\Gamma_{0}$, at a blow-up time $T$. It is known [21] that the classical solution exists and is unique locally in time. Recently, in [14], it became clear that this model could be interpreted as a particular case of the abstract Cauchy problem; thus, the classical solvability (locally in time) may be proved using the nonlinear abstract Cauchy-Kovalevskaya Theorem. We also mention here that the problem is Hadamard well-posed in our case [3].

The problem of estimating $T$ is of primary importance. One of the "folklore" questions is the lifetime of the starlike dynamics in a Hele-Shaw cell. The majority of investigators believe that this lifetime is infinite in the case of a starlike initial domain whereas some of them do not. Our aim is to settle this question. A domain $\Omega \subset \mathbb{C}, 0 \in \Omega$, is said to be starlike (with respect to the origin) if each ray starting at the origin intersects $\Omega$ in a set that is either a line segment or a full ray. If a function $f(\zeta)$ maps $U$ onto a domain that is starlike, $f(0)=0$, then we say that $f(\zeta)$ is a starlike function. We denote the class of starlike functions by $S^{*}$. A criterion for a function $f(\zeta), \zeta \in U, f(0)=0, f^{\prime}(0)>0$, to be starlike is the following inequality:

$$
\operatorname{Re} \frac{\zeta f^{\prime}(\zeta)}{f(\zeta)}>0, \quad \zeta \in U
$$

This standard criterion can be found, e.g., in [5, 2, 7, 13].

We prove rigorously (Theorem 3.4) that, starting with a smooth analytic starlike phase domain $\Omega_{0}$, the Hele-Shaw chain of subordinating domains $\Omega(t)$ exists as a classical solution for an infinite time under injection.

\section{Monotone Change of STROng $\alpha$-STARLiKEnESS}

The class $S^{*}$ is a union of classes $S_{\alpha}^{*}$ of so-called strongly starlike functions of order $\alpha, 0<\alpha \leq 1$, defined by D. A. Brannan and W. E. Kirwan [1] and J. Stankiewicz [19]. A function $f: U \rightarrow \mathbb{C}, f(0)=0, f^{\prime}(0)>0$, is said to be from $S_{\alpha}^{*}$ if for all $\zeta \in U$,

$$
\left|\arg \frac{\zeta f^{\prime}(\zeta)}{f(\zeta)}\right|<\alpha \frac{\pi}{2} .
$$

This class of functions is characterized as follows. Every level line $f\left(r e^{i \theta}\right), \theta \in$ $[0,2 \pi), f \in S_{\alpha}^{*}$, is reachable from outside by the radial angle $\pi(1-\alpha)$. We will call the images of the unit disk under functions from $S_{\alpha}^{*}$ strongly starlike of order $\alpha$ as well. Clearly, $S^{*}=S_{1}^{*}$.

We prove that, starting with a phase domain $\Omega_{0}$ that is strongly starlike of order $\alpha$ and bounded by an analytic curve, we obtain a subordination chain of domains $\Omega(t)$ (and functions $f(\zeta, t)$ ) strongly starlike of order $\alpha(t)$ with a decreasing order $\alpha(t)$.

Theorem 2.1. Let $f_{0} \in S_{\alpha}^{*}, \alpha \in(0,1]$, be analytic and univalent in a neighbourhood of $\bar{U}$. Then the classical solution $f(\zeta, t)$ to the Polubarinova-Galin equation (1.1) forms a subordination chain of strongly starlike functions of order $\alpha(t)$ with a strictly decreasing $\alpha(t)$ during the time of existence.

Proof. Let $T$ be such that the classical solution $f(\zeta, t)$ exists during the time $t \in$ $[0, T), T>0$. Since all functions $f(\zeta, t)$ have analytic univalent extension into a neighbourhood of $\bar{U}$ during the time of the existence of the classical solution to 
(1.1), their derivatives $f^{\prime}(\zeta, t)$ are continuous and do not vanish in $\bar{U}$. Moreover, $f(\zeta, t)$ are starlike in $U$ (see [8, 20]). Therefore, there exists $\alpha(t), 0<\alpha(t) \leq 1$, such that $f(\zeta, t) \in S_{\alpha(t)}^{*}$ and $f(\zeta, t) \notin S_{\alpha(t)-\varepsilon}^{*}$ for any $\varepsilon>0$.

Let us fix $t_{0} \in[0, T)$ and consider the set $A$ of all points $\zeta,|\zeta|=1$, for which $\left|\arg \frac{\zeta f^{\prime}\left(\zeta, t_{0}\right)}{f\left(\zeta, t_{0}\right)}\right|=\alpha \pi / 2$. First, we deal with the subset $A^{+}$of $A$ where

$$
\arg \frac{\zeta f^{\prime}\left(\zeta, t_{0}\right)}{f\left(\zeta, t_{0}\right)}=\frac{\alpha \pi}{2} .
$$

The sets $A^{+}$and $A^{-}=A \backslash A^{+}$are closed and do not intersect. One of the sets $A^{+}$ and $A^{-}$is allowed to be empty. Without loss of generality, we suppose that $A^{+} \neq$ $\varnothing$. For any point $\zeta \in A^{+}$, we have

$$
\operatorname{Im} \frac{\zeta f^{\prime}\left(\zeta, t_{0}\right)}{f\left(\zeta, t_{0}\right)}>0
$$

The argument $\arg \frac{\zeta f^{\prime}\left(\zeta, t_{0}\right)}{f\left(\zeta, t_{0}\right)}$ attains its maximum on $\zeta \in \partial U$ at the points of $A^{+}$. Therefore,

$$
\frac{\partial}{\partial \theta} \arg \frac{e^{i \theta} f^{\prime}\left(e^{i \theta}, t_{0}\right)}{f\left(e^{i \theta}, t_{0}\right)}=0, \quad \zeta=e^{i \theta} \in A^{+} .
$$

The argument $\arg \frac{r e^{i \theta} f^{\prime}\left(r e^{i \theta}, t_{0}\right)}{f\left(r e^{i \theta}, t_{0}\right)}, e^{i \theta} \in A^{+}$, attains its maximum on $r \in[0,1]$ at $r=1$. Hence,

$$
\left.\frac{\partial}{\partial r} \arg \frac{r e^{i \theta} f^{\prime}\left(r e^{i \theta}, t_{0}\right)}{f\left(r e^{i \theta}, t_{0}\right)}\right|_{r=1} \geq 0 .
$$

We calculate

$$
\begin{aligned}
& \operatorname{Re}\left[1+\frac{\zeta f^{\prime \prime}\left(\zeta, t_{0}\right)}{f^{\prime}\left(\zeta, t_{0}\right)}-\frac{\zeta f^{\prime}\left(\zeta, t_{0}\right)}{f\left(\zeta, t_{0}\right)}\right]=0, \\
& \operatorname{Im}\left[1+\frac{\zeta f^{\prime \prime}\left(\zeta, t_{0}\right)}{f^{\prime}\left(\zeta, t_{0}\right)}-\frac{\zeta f^{\prime}\left(\zeta, t_{0}\right)}{f\left(\zeta, t_{0}\right)}\right] \geq 0,
\end{aligned}
$$

where $\zeta \in A^{+}$.

Let us represent the derivative

$$
\frac{\partial}{\partial t} \arg \frac{\zeta f^{\prime}(\zeta, t)}{f(\zeta, t)}=\operatorname{Im} \frac{\partial}{\partial t} \log \frac{f^{\prime}(\zeta, t)}{f(\zeta, t)}=\operatorname{Im}\left(\frac{\frac{\partial}{\partial t} f^{\prime}(\zeta, t)}{f^{\prime}(\zeta, t)}-\frac{\frac{\partial}{\partial t} f(\zeta, t)}{f(\zeta, t)}\right) .
$$

Now we differentiate the Polubarinova-Galin equation (1.1) with respect to $\theta$ as

$$
\operatorname{Im}\left(\overline{f^{\prime}(\zeta, t)} \frac{\partial}{\partial t} f^{\prime}(\zeta, t)-\overline{\zeta f^{\prime}(\zeta, t)} \dot{f}(\zeta, t)-\overline{\zeta^{2} f^{\prime \prime}(\zeta, t)} \dot{f}(\zeta, t)\right)=0, \quad \zeta=e^{i \theta}
$$

This equality is equivalent to

$$
\begin{aligned}
& \left|f^{\prime}(\zeta, t)\right|^{2} \operatorname{Im}\left(\frac{\frac{\partial}{\partial t} f^{\prime}(\zeta, t)}{f^{\prime}(\zeta, t)}-\frac{\frac{\partial}{\partial t} f(\zeta, t)}{f(\zeta, t)}\right) \\
& \quad=\operatorname{Im}\left[\overline{\zeta f^{\prime}(\zeta, t)} \dot{f}(\zeta, t)\left(\overline{\left(\frac{\zeta f^{\prime \prime}(\zeta, t)}{f^{\prime}(\zeta, t)}\right)}-\frac{\zeta f^{\prime}(\zeta, t)}{f(\zeta, t)}+1\right)\right] .
\end{aligned}
$$


Substituting (1.1) and (2.4) in the latter expression, we have

$$
\left.\frac{\partial}{\partial t} \arg \frac{\zeta f^{\prime}(\zeta, t)}{f(\zeta, t)}\right|_{\zeta \in A^{+}, t=t_{0}}=\frac{-1}{\left|f^{\prime}\left(\zeta, t_{0}\right)\right|^{2}} \operatorname{Im}\left(\frac{\zeta f^{\prime}\left(\zeta, t_{0}\right)}{f\left(\zeta, t_{0}\right)}+\frac{\zeta f^{\prime \prime}\left(\zeta, t_{0}\right)}{f^{\prime}\left(\zeta, t_{0}\right)}\right) .
$$

The right-hand side of this equality is continuous on $A^{+}$and strictly negative because of (2.3) and (2.5). Therefore,

$$
\left.\max _{\zeta \in A^{+}} \frac{\partial}{\partial t} \arg \frac{\zeta f^{\prime}(\zeta, t)}{f(\zeta, t)}\right|_{t=t_{0}}=-\delta<0 .
$$

There exists a neighborhood $A^{+}(\delta)$ on the unit circle of $A^{+}$such that $A^{+}(\delta)$ and $A^{-}$do not intersect and

$$
\left.\frac{\partial}{\partial t} \arg \frac{\zeta f^{\prime}(\zeta, t)}{f(\zeta, t)}\right|_{\zeta \in A^{+}(\delta), t=t_{0}}<-\frac{\delta}{2} .
$$

There is a positive number $\sigma$ such that

$$
\left.\max _{\zeta \in \partial U \backslash A^{+}(\delta)} \arg \frac{\zeta f^{\prime}(\zeta, t)}{f(\zeta, t)}\right|_{t=t_{0}}=\frac{\alpha \pi}{2}-\sigma .
$$

We choose such $s>0$ that

(i) $t_{0}+s<T$;

(ii) $\left.\frac{\partial}{\partial t} \arg \frac{\zeta f^{\prime}(\zeta, t)}{f(\zeta, t)}\right|_{\zeta \in A^{+}(\delta)}<0, \quad t \in\left[t_{0}, t_{0}+s\right]$;

(iii) $\max _{\zeta \in \partial U \backslash A^{+}(\delta)} \arg \frac{\zeta f^{\prime}(\zeta, t)}{f(\zeta, t)} \leq \frac{\alpha \pi}{2}-\frac{\sigma}{2}, \quad t \in\left[t_{0}, t_{0}+s\right]$.

The condition (ii) implies that

$$
\arg \frac{\zeta f^{\prime}(\zeta, t)}{f(\zeta, t)}<\frac{\alpha \pi}{2}, \quad t \in\left(t_{0}, t_{0}+s\right], \zeta \in A^{+}(\delta) .
$$

Thus, the condition (iii) yields

$$
\alpha^{+}(t):=\max _{\zeta \in \partial U} \arg \frac{\zeta f^{\prime}(\zeta, t)}{f(\zeta, t)}<\frac{\alpha \pi}{2}=\alpha\left(t_{0}\right), \quad \text { for all } t \in\left(t_{0}, t_{0}+s\right] .
$$

This means that $\alpha^{+}(t)$ is strictly decreasing in $[0, T)$.

If the set $A^{-} \neq \varnothing$, then we can define the function

$$
\alpha^{-}(t):=-\min _{\zeta \in \partial U} \arg \frac{\zeta f^{\prime}(\zeta, t)}{f(\zeta, t)} .
$$

Similar argumentation shows that $\alpha^{-}(t)$ is strictly decreasing.

If $A^{-}=\varnothing\left(\right.$ or $\left.A^{+}=\varnothing\right)$, then $\alpha(t)=\alpha^{+}(t)$ (or $\left.=\alpha^{-}(t)\right)$ for $t \in\left[t_{0}, t_{0}+s\right], s$ sufficiently small.

We set the function $\alpha(t)=\max \left\{\alpha^{+}(t), \alpha^{-}(t)\right\}$ in the case $A^{+} \neq \varnothing$ and $A^{-} \neq$ $\varnothing$. This function $\alpha(t)$ is strictly decreasing, and the proof is complete.

We remark that a similar result, with estimates of $\alpha(t)$, has been independently obtained by O. Kuznetsova [10]. 


\section{INFINITE LIFETIME}

In this section first we will prove that if the classical solution to (1.1) exists during the time interval $[0, T)$, then the limiting function $\lim _{t \rightarrow T-0} f(\zeta, t) \equiv f(\zeta, T)$ is analytic in some neighbourhood of the unit disk $U$. Here the limit is taken with respect to the uniform convergence on compacts of the unit disk $U$. It exists because $f(\zeta, t)$ is a subordination chain, and due to the Carathéodory Kernel Theorem. Then we will obtain the main result about the infinite lifetime.

Lemma 3.1. Let the classical solution to (1.1) exist during the time interval $[0, T)$, $0<T<\infty, \Omega(t)=f(U, t)$, and let the initial function $f(\zeta, 0)$ be analytic and univalent in a neighbourhood of the closure of the unit disk $U$. Then, there is $\eta>0$ such that the function $f(\zeta, t)$ is analytic in $U_{1+\eta}=\{\zeta:|\zeta|<1+\eta\}$ for all $t \in[0, T]$, univalent in $U$, and possibly $f(\zeta, T)$ has a vanishing derivative at some points of the unit circle $\partial U$ or is not univalent on $\partial U$. It follows that $\Omega(T) \equiv f(U, T)$ is a simply connected domain with an analytic boundary $\Gamma(T)=\partial \Omega(T)$ with possible analytic singularities in the form of finitely many cusps and double points.

Proof. By the Carathéodory Kernel Theorem the domain

$$
\Omega(T)=\bigcup_{t \in[0, T)} \Omega(t)
$$

is just the same as in the formulation of the lemma, and $\Omega(T)$ is a simply connected domain.

It is well known (see, e.g., [6, 15, 16]) and easily verified directly that for any analytic and integrable function $\Phi$ in $\Omega(T)$ the identity

$$
\iint_{\Omega(t)} \Phi(z) d \sigma_{z}=\iint_{\Omega(0)} \Phi(z) d \sigma_{z}+2 \pi t \Phi(0)
$$

holds for $t \in[0, T)$. We note also that since the normal velocity on the boundary never vanishes, we have the strict monotonicity of the subordination chain of domains:

$$
\overline{\Omega(s)} \subset \Omega(t) \quad \text { for } s<t \text { and } s, t \in(0, T) .
$$

Letting $t \rightarrow T$, we see that (3.1) and (3.2) hold for $t=T$, i.e., for $\Omega(T)$ as well.

In order to give a rigorous proof of the statement about the properties of $f(\zeta, T)$ we analytically extend the mapping $f(\zeta, T)$ following the scheme proposed in [6] Theorem 9]; see also [17, Theorem 1.7]. Let us choose the function $\Phi(z)=(z-w)^{-1}$ for $w \notin \Omega(t), t \in(0, T]$, in (3.1). This gives

$$
\hat{\chi}_{\Omega(t)}=\hat{\chi}_{\Omega(0)}+2 \pi t \hat{\delta}
$$

where

$$
\hat{\mu}(w)=-\frac{1}{\pi} \iint_{\Omega(t)} \frac{d \mu(z)}{z-w}
$$

denotes the Cauchy transformation of a measure $\mu$, normalized so that $\partial \hat{\mu} / \partial \bar{w}=$ $\mu(w), \chi$ stands for the characteristic function of the corresponding domain, and $\delta$ is the Dirac distribution. 
We define a function $S(z, t)$ by

$$
S(z, t)=\bar{z}-\hat{\chi}_{\Omega(t)}(z)+\hat{\chi}_{\Omega(0)}(z)+\frac{2 t}{z}
$$

for $z \in \overline{\Omega(t)} \backslash \overline{\Omega(0)}$, for each $t \in(0, T]$. Then $S(z, t)$ as a function of $z$ is continuous in $\overline{\Omega(t)} \backslash \overline{\Omega(0)}$, analytic in $\Omega(t) \backslash \overline{\Omega(0)}$ (one easily calculates that $\partial S / \partial \bar{z}=0$ ), and $S(z, t)=\bar{z}$ on $\Gamma(t)=\partial \Omega(t)$ by (3.3). Thus, $S(z, t)$ is a one-sided Schwarz function of $\Gamma(t)$ [16, 18]. The transformation $z \mapsto \overline{S(z, t)}$ is a one-sided reflection over $\Gamma(t)$, which we will use to extend $f(\zeta, t), t \in(0, T]$. Let us fix $t \in(0, T]$. We set $r(t)=\max _{z \in \partial \Omega(0)}\left|f^{-1}(z, t)\right|$ and consider a point $\zeta, r(t)<|\zeta|<1$. The point $1 / \bar{\zeta}$ is its reflection through $\partial U$.

We define the function $f(\zeta, t)$ in a neighbourhood of the unit circle $1<|\zeta|<$ $1 / r(t)$ outside $U$ by $f(1 / \bar{\zeta}, t) \equiv \overline{S(f(\zeta, t), t)}$. This defines $f$ analytically in the annulus $1<|\zeta|<1 / r(t)$. Across $\partial U$ we have a certain form of continuity because of the continuity of $S(z, t)$. Indeed, as $|\zeta| \rightarrow 1$ with $\zeta \in U$ we have

$$
|f(\zeta, t)-f(1 / \bar{\zeta}, t)|=|f(\zeta, t)-\overline{S(f(\zeta, t), t)}| \rightarrow 0,
$$

where $z=\overline{S(z, t)}$ on $\Gamma(t)$, and therefore, given $\varepsilon>0$, we have $|z-\overline{S(z, t)}|<\varepsilon$ for $z \in \Omega(t)$ in some neighbourhood of $\Gamma(t)$. By now the function $f(\zeta, t)$ is defined in $U$ as well as in the annulus $1<|\zeta|<1 / r(t)$, and hence, almost everywhere in the disk $|\zeta|<1 / r(t)$. Let us prove that the distributional derivative $\partial f(\zeta, t) / \partial \bar{\zeta}$ vanishes in $|\zeta|<1 / r(t)$, using (3.4). Obviously, we must verify this across the circle $|\zeta|=1$. Given a test function $\varphi$ with compact support in $|\zeta|<1 / r(t)$, we have

$$
\begin{aligned}
\left\langle\frac{\partial f}{\partial \bar{\zeta}}, \varphi\right\rangle & =-\iint_{\mathbb{C}} f(\zeta, t) \frac{\partial \varphi}{\partial \bar{\zeta}} d \sigma_{\zeta} \\
& =-\frac{1}{2 i} \iint_{U} f(\zeta, t) \frac{\partial \varphi}{\partial \bar{\zeta}} d \bar{\zeta} d \zeta-\frac{1}{2 i} \iint_{|\zeta|>1} f(\zeta, t) \frac{\partial \varphi}{\partial \bar{\zeta}} d \bar{\zeta} d \zeta \\
& =-\frac{1}{2 i} \lim _{\varepsilon \downarrow 0}\left(\int_{|\zeta|=1-\varepsilon} f(\zeta, t) \varphi(\zeta) d \zeta-\int_{|\zeta|=1+\varepsilon} f(\zeta, t) \varphi(\zeta) d \zeta\right) \\
& =-\frac{1}{2 i} \lim _{\varepsilon \downarrow 0} \int_{|\zeta|=1-\varepsilon}(f(\zeta, t)-f(1 / \bar{\zeta}, t)) \varphi(\zeta) d \zeta=0 .
\end{aligned}
$$

In the above curve integrals we take the counterclockwise direction on the circles. Thus, the function $f(\zeta, t)$ is analytic in the disk $|\zeta|<1 / r(t)$.

For any pair of numbers $s, t$ such that $0<s<t \leq T$, we have that the function $h(\zeta, s, t) \equiv f^{-1}(f(\zeta, s), t)$ maps the unit disk into itself and $h(0, s, t) \equiv 0$. A simple application of the Schwarz Lemma to the function $h$ shows that

$$
f^{-1}(\Omega(0), t) \subset U_{r(s)} .
$$

Therefore, $r(t) \leq r(s)$. For a sufficiently small $\varepsilon>0$ we choose $\delta$ such that the solution $f(\zeta, t)$ is analytic in the disk $U_{1+\varepsilon}$ for any $t \in[0, \delta)$. Then we define

$$
\eta=\min \left(\varepsilon, \frac{1}{r(\delta)}-1\right) \text {. }
$$


Thus, we have defined the function $f(\zeta, t)$ in the disk $U_{1+\eta}$ for all $t \in[0, T]$. To get a pointwise definition of $f$ on the circle $|\zeta|=1$ one may use the Cauchy integral formula for the disk $|\zeta|<1+\eta / 2$. This finishes the proof of the lemma.

Lemma 3.2. Let $f_{0} \in S^{*}$ be analytic and univalent in a neighbourhood of the closure $\bar{U}$ of the unit disk $U$. If the solution $f(\zeta, t)$ to the Polubarinova-Galin equation (1.1) exists during the time interval $[0, T)$, then it forms a subordination chain of starlike functions such that the limiting domain $\Omega(T)$ has a smooth analytic boundary.

Proof. By the assumptions on $f_{0}$ the initial domain $\Omega(0)$ has a smooth analytic boundary and the solution $f(\zeta, t)$ to (1.1) exists locally in time $t \in[0, s)$. The function $f_{0} \in S_{\alpha}^{*}$ for some $\alpha \in(0,1]$. The function $f(\zeta, t)$ belongs to the class $S_{\alpha(t)}^{*}$ with $\alpha(t)<1$ for any $t \in(0, s)$, due to Theorem 2.1 Therefore, we can prove Lemma 3.2 choosing $f(\zeta, t)$ with some $t \in(0, s)$ as an initial mapping, $f(\zeta, t) \in S_{\alpha(t)}^{*}$ with $\alpha(t)<1$. We prove that $\partial \Omega(T)$ does not contain a cusp or a double point on its boundary.

Define the limiting function $f(\zeta, T)=\lim _{t \rightarrow T-0} f(\zeta, t)$, where the limit is taken locally uniformly in $U$. The function $f(\zeta, T)$ is univalent, strongly starlike of order $\alpha(T)=\lim _{t \rightarrow T-0} \alpha(t)<1$, and has a continuous extension on $\bar{U}$. According to the geometric characterization of the class $S_{\alpha(T)}^{*}$, the boundary of the domain $\Omega(T)=$ $f(U, T)$ is reachable by the radial external angles $\pi(1-\alpha)$, which implies that there is no cusp or double point on the boundary of $\Omega(T)$. This completes the proof.

Lemma 3.3. Let $f(\zeta, t)$ be the classical solution to the equation (1.1) that exists during the time interval $[0, T)$ with a starlike initial mapping $f_{0}$ as in Lemma 3.2. Then, there exists $\varepsilon>0$ such that the classical solution exists during the time interval $[0, T+\varepsilon)$.

Proof. The limiting domain $\Omega(T)$ is simply connected and has an analytic boundary. The limiting mapping $f(\zeta, T)$ is analytic in a neighbourhood of $\bar{U}$ by Lemma 3.1 and strongly starlike of order $\alpha(T)<1$ by Lemma 3.2 Therefore, there exists an $\eta>0$ such that $f(\zeta, T)$ is starlike and univalent in the disk $|\zeta|<1+\eta$. Let us construct the subordination chain of mappings $f_{2}(\zeta, t)$ satisfying the PolubarinovaGalin equation (1.1) with the initial data $f_{2}(\zeta, 0) \equiv f(\zeta, T)$. The classical solution exists and is unique locally in time, say $t \in[0, \varepsilon)$. Moreover, we have

$$
\lim _{t \rightarrow T-0} f(\zeta, t)=\lim _{t \rightarrow 0+0} f_{2}(\zeta, t)=f(\zeta, T)
$$

and

$$
\lim _{t \rightarrow T-0} f^{\prime}(\zeta, t)=\lim _{t \rightarrow 0+0} f_{2}^{\prime}(\zeta, t)=f^{\prime}(\zeta, T)
$$

locally uniformly in $U_{1+\eta}$. We rewrite the equation (1.1) in $U$ using the Schwarz kernel as

$$
\dot{f}(\zeta, t)=\zeta f^{\prime}(\zeta, t) \frac{1}{2 \pi} \int_{0}^{2 \pi} \frac{1}{\left|f^{\prime}\left(e^{i \theta}, t\right)\right|^{2}} \frac{e^{i \theta}+\zeta}{e^{i \theta}-\zeta} d \theta, \quad t \in[0, T),|\zeta|<1 .
$$

A similar equation is valid for the chain $f_{2}(\zeta, t)$ in the time interval $[0, \varepsilon)$. Taking the limit in the above equation as $t \rightarrow T-0$ we observe that there exists the one-sided limit $\dot{f}(\zeta, T-0)$. Similarly, the one-sided limit $\dot{f}_{2}(\zeta, 0+0)$ exists, and 
they are equal. Let us define $f(\zeta, t) \equiv f_{2}(\zeta, t-T)$ in the interval $t \in[T, T+\varepsilon)$. The above observations yield that the function thus extended is continuous in the interval $t \in[0, T+\varepsilon)$, analytic, univalent and starlike in some neighbourhood of $\bar{U}$. Moreover, it is differentiable at the point $t=T$, and being extended onto the unit circle, satisfies the equation (1.1). Thus, it is a unique classical solution in the interval $t \in[0, T+\varepsilon)$, and the lemma is proved.

Theorem 3.4. Starting with a starlike phase domain $\Omega_{0}$ with an analytic boundary, the lifetime of the classical Hele-Shaw starlike dynamics $\Omega(t)$ is infinite.

Proof. Indeed, if the classical solution exists during the finite interval $t \in[0, T)$ and does not exist in $t \in[T, T+\varepsilon)$ for any $\varepsilon>0$, then this contradicts Lemma 3.3

\section{REFERENCES}

[1] D. A. Brannan and W. E. Kirwan, On some classes of bounded univalent functions, J. London Math. Soc. (2), 1 (1969), 431-443. MR 40:4439

[2] P. Duren, Univalent functions, Grundlehren der Mathematischen Wissenschaften, Band 259, Springer-Verlag, New York, 1983. MR 85j:30034

[3] C. M. Elliott and V. Janovsky, A variational inequality approach to Hele-Shaw flow with a moving boundary, Proc. Roy. Soc. Edinburgh, A88 (1981), 93-107. MR 82d:76031

[4] L. A. Galin, Unsteady filtration with a free surface, C. R. (Dokl.) Acad. Sci. URSS, 47 (1945), 246-249. MR 7:229b

[5] G. M. Goluzin, Geometric theory of functions of a complex variable, Transl. Math. Monogr., Vol. 26, American Mathematical Society, Providence, RI, 1969. MR 40:308

[6] B. Gustafsson, Applications of variational inequalities to a moving boundary problem for Hele-Shaw flows, SIAM J. Math. Analysis, 16 (1985), no. 2, 279-300. MR 86m:35155

[7] A. W. Goodman, Univalent functions, Vols. I, II, Mariner Publishing Company, Inc., U. South Florida, Tampa, FL, 1983.

[8] Yu. E. Hohlov, D. V. Prokhorov, and A. Vasil'ev, On geometrical properties of free boundaries in the Hele-Shaw flow moving boundary problem, Lobachevskiü J. Math., 1 (1998), 3-13 (electronic). MR 99m:76054

[9] S. D. Howison, Complex variable methods in Hele-Shaw moving boundary problems, European J. Appl. Math., 3 (1992), no. 3, 209-224. MR 94f:76025

[10] O. Kuznetsova, Invariant families in the Hele-Shaw problem, Preprint TRITA-MAT2003-07, Royal Institute of Technology, Stockholm, Sweden, 2003.

[11] P. Ya. Polubarinova-Kochina, On a problem of the motion of the contour of a petroleum shell, Dokl. Akad. Nauk USSR, 47 (1945), no. 4, 254-257 (in Russian); English transl., On the displacement of the oil-bearing contour, C. R. (Dokl.) Acad. Sci. URSS, 47 (1945), 250-254. MR 7:140i

[12] P. Ya. Polubarinova-Kochina, Concerning unsteady motions in the theory of filtration, Prikl. Matem. Mech., 9 (1945), no. 1, 79-90. (in Russian; English summary). MR 7:95d

[13] Ch. Pommerenke, Univalent functions, with a chapter on quadratic differentials by G. Jensen, Vandenhoeck \& Ruprecht, Göttingen, 1975. MR 58:22526

[14] M. Reissig and L. von Wolfersdorf, A simplified proof for a moving boundary problem for Hele-Shaw flows in the plane, Ark. Mat., 31 (1993), no. 1, 101-116. MR 94m:35250

[15] S. Richardson, Hele-Shaw flows with a free boundary produced by the injection of fluid into a narrow channel, J. Fluid Mech. 56 (1972), 609-618.

[16] M. Sakai, Quadrature domains, Lecture Notes in Math. 934, Springer-Verlag, New York, 1982. MR 84h:41047

[17] M. Sakai, Regularity of boundaries of quadrature domains in two dimensions, SIAM J. Math. Analysis 24 (1993), no. 2, 341-364. MR 94c:30054

[18] H. S. Shapiro, The Schwarz function and its generalization to higher dimensions, University of Arkansas, Lecture Notes in Mathematical Sciences, Vol. 9, John Wiley and Sons, New York, 1992. MR 93g:30059

[19] J. Stankiewicz, Some remarks concerning starlike functions, Bull. Acad. Polon. Sci., Sér. Sci. Math. Astronom. Phys., 18 (1970), 143-146. MR 41:8650 
[20] A. Vasil'ev, Univalent functions in the dynamics of viscous flows, Comput. Methods and Function Theory 1 (2001), no. 2, 311-337.

[21] Yu. P. Vinogradov and P. P. Kufarev, On a problem of filtration, Akad. Nauk SSSR. Prikl. Mat. Meh., 12 (1948), 181-198 (in Russian). MR 9:540j]

Department of Mathematics, Royal Institute of Technology, Stockholm 100 44, SweDEN

E-mail address: gbjorn@math.kth.se

Department of Mathematics and Mechanics, Saratov State University, Saratov 410012, RUSSIA

E-mail address: ProkhorovDV@info.sgu.ru

Departamento de Matemática, Universidad Técnica Federico Santa María, Casilla 110-V, Valparaíso, Chile

E-mail address: alexander.vasiliev@mat.utfsm.cl 